\title{
Digital Era in Cross-Sectional Human Anatomy: The Use of Detailed Images in the Study Process
}

\author{
Dzintra Kazoka, Mara Pilmane \\ Rìga Stradinš University
}

\section{INTRODUCTION}

An increasing number of digital technologies have been introduced which assist in some practical aspects of the teaching and learning of Human Anatomy. For modern-day students, more and more cross-sectional images of the human body are available as materials in their studies. Digital 2D images and/or 3D images such as high-resolution computed tomography (CT) scans and magnetic resonance (MR) have been received with much enthusiasm and have found their way into anatomy courses and practical classes. An emphasis on sectional anatomy can help medical students develop and improve their knowledge of cross sectional imaging.

The aim of this study was to assess and verify the impact of cross-sectional images on the study of Human Anatomy.

\section{MATERIALS AND METHODS}

200 medical students in their 2nd study year at Rīga Stradinš University were included in this investigation in 2018. In practical classes, all students were asked by tutors to identify several anatomical structures using "Anatomage", a 3D virtual dissection table (USA). They were randomly divided into two groups: Group I analysed cross-sectional images of the human body after cutting and segmentation with interactive tools; Group II studied X-rays, CT scans and MRI images of different regions and systems. Similar images in all possible cross-sectional planes were displayed. The pathological findings from the cross-sectional images were used to develop several clinical questions and cases. At the beginning of each practical class, the tutor spent about 30 minutes demonstrating the themes in the images to all the students. At the end of the teaching session, their understanding of the relationships between anatomical structures and the rate of cross-sectional image effectiveness among both groups was assessed by means of discussion.

\section{RESULTS}

By this time, students were able to identify the position of bones and organs from the lectures, practical classes and dissections. The majority of students recognized the landmarks, anatomical structures and relationships between them on cross-sectional images in three planes. By removing different kinds of tissue, students in both groups were able to learn more difficult anatomical topics and to explain details in these complicated regions. Self-assessment exercises helped students verify their understanding of topographical relations and regional anatomy. In these groups, students with less prior anatomy experience wanted to focus more on the basics of anatomy whereas students with more prior anatomy experience wanted to see and study more complex and interactive materials in cross-sectional images.

\section{CONCLUSION}

This study demonstrated the role of the 3D virtual dissection table "Anatomage" in facilitating students' ability to interpret crosssectional images and understand the relationships between different anatomical structures. All the X-rays, CT scans and MRI cross-sectional images used played an important role in the teaching and learning of Human Anatomy. Skills and the ability to interpret these medical images is important for the application of anatomical knowledge right from basic studies until clinical courses.

\section{KEYWORDS}

Anatomage, human anatomy, cross-sectional images, students 